\title{
Le LHC dans sa dernière ligne droite...
}

\author{
Laurent Serin \\ Laboratoire de l'Accélérateur Linéaire, Université Paris 11, Orsay
}

Déjà émise au début des années 80 , lors de la conception du LEP au CERN avec son tunnel de $27 \mathrm{~km}$ de circonférence, l'idée du LHC et des détecteurs associés a réellement commencé à prendre corps au début des années 90. Devant l'ampleur des difficultés techniques, tant sur l'accélérateur que sur les moyens de détection dans un environnement à fortes radiations, presque dix années de recherche et de développement furent nécessaires avant que le concept n'aboutisse à la construction et, depuis plusieurs mois, à l'installation de la machine et de ses détecteurs.

Le LHC comporte des aimants supraconducteurs (plus de 1200 dipôles) qui, après quelques difficultés, ont atteint leurs performances nominales et permettront d'obtenir une énergie de 14 téraélectronvolt ( $\mathrm{TeV}$ ) dans le centre de masse des collisions proton-proton (actuellement l'énergie la plus importante disponible est de $2 \mathrm{TeV}$ au Tévatron au Fermilab à Chicago). Le refroidissement de ces aimants à 1,9 kelvin fera du LHC la plus grande installation cryogénique au monde. La photographie 1 montre la disponibilité des différents éléments que l'on peut apercevoir actuellement un peu partout au CERN. Sous la houlette du directeur général, Robert Aymar, tous les efforts sont actuellement déployés pour que l'ensemble des éléments du LHC soient installés avant l'été 2007 et que les premières collisions aient lieu avant 2008.

L'un des objectifs principaux de la physique au LHC est la recherche du boson de Higgs ou de tout autre signe de nouveaux phénomènes physiques apparaissant au-delà du TeV. Dans ce but, deux expériences généralistes, Atlas et CMS, grandes par leur taille $(\sim 40 \times 20 \times 20 \mathrm{~m})$ et par leur nombre de participants (environ 1500 dans chacune), ont été conçues $^{1}$. Après une longue période de construction des sous-détecteurs et de leurs électroniques dans différents instituts de par le monde, et en particulier dans les laboratoires de I'IN2P3/CNRS et du DAPNIA/CEA, l'activité est désormais concentrée au CERN avec l'installation définitive des détecteurs et leur mise en fonctionnement pour les premières collisions. Les photographies 2 et 3 illustrent bien l'avancement de la réalisation de l'expérience Atlas sur les trois dernières années. En mécanique comme en électronique, les techniques ont parfois dû être poussées à leurs limites, mais l'ingéniosité des équipes va permettre de fournir des détecteurs satisfaisant les exigences de performance. Le prochain grand défi sera ensuite d'analyser les petabytes de données ${ }^{2}$ qui vont s'accumuler, ce pour quoi les moyens informatiques (puissance, réseau, stockage...) devront être à la hauteur des investissements consentis. Sous certaines hypothèses, seule une collision sur $10^{12}$ produira une désintégration de la particule de Higgs, donc mieux vaut ne pas la rater !

\section{Lexique des sigles}

LEP : Large Electron Positron collider (anneau de collision électron-positron).

CERN : Centre Européen pour la Recherche Nucléaire (souvent désormais écrit Centre Européen pour la Physique des Particules).

DAPNIA : Département d'Astrophysique, de physique des Particules, de physique Nucléaire et de l'Instrumentation Associée (du CEA/Saclay).

LHC : Large Hadron Collider (anneau de collisions de hadrons).

ATLAS : A Toroid Lhc ApparatuS.

CMS : Compact Muon Solenoid.

ALICE : A Large Ion Collider Experiment.

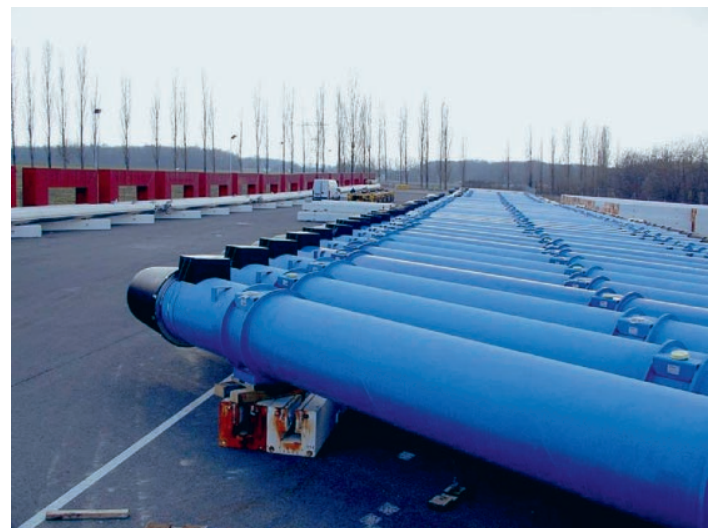

Figure 1: Vue des dipôles sur un parking du CERN, en attente d'être descendus dans le tunnel du LHC.

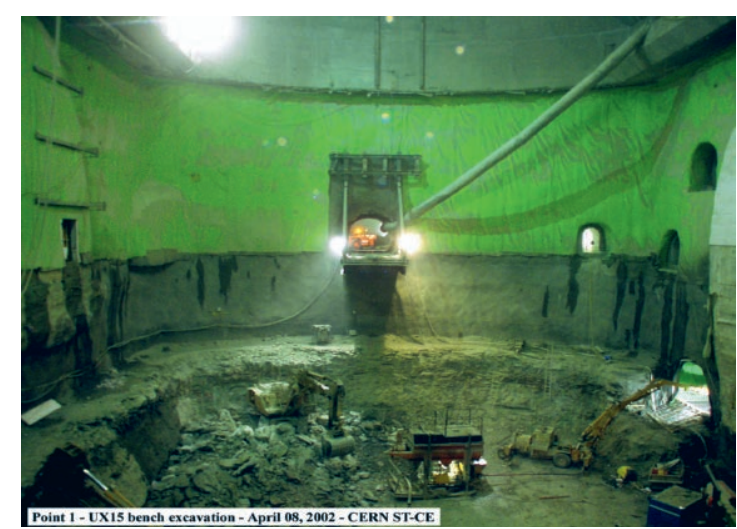

Figure 2 : La caverne devant accueillir le détecteur de l'expérience Atlas, telle qu'elle se présentait en avril 2002.

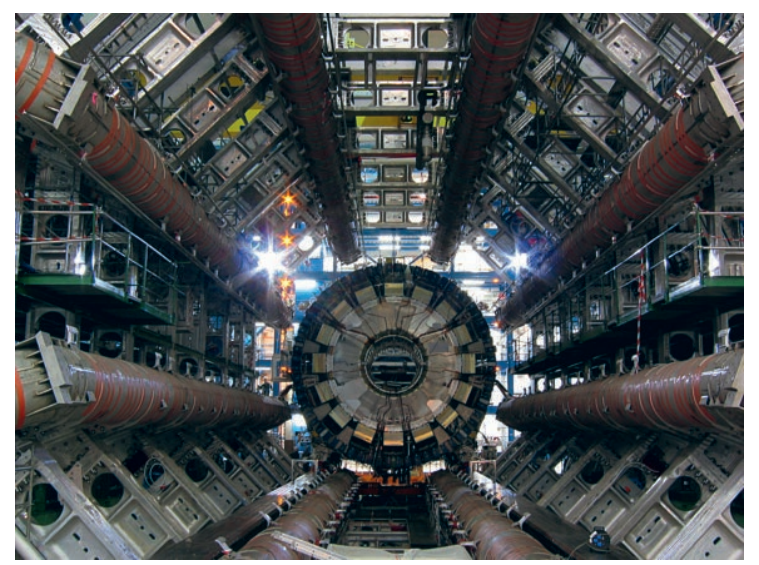

Figure 3 : La même caverne majoritairement remplie en novembre 2005. Au centre on voit le calorimètre central dans lequel les groupes français ont eu une très forte implication. À la circonférence, les huit bobines supraconductrices de 25 m de long, permettant d'obtenir un champ toroïdal. Ce système toroïdal a été proposé et conçu par le CEA. Sur la bobine au fond à gauche, une silhouette humaine est visible, montrant le gigantisme du détecteur!

1 II existe aussi deux autres expériences prévues auprès du LHC, dans lesquelles la communauté française est fortement impliquée : LHCb, dédiée à l'étude des mésons "beaux ", et Alice, dédiée à celle des collisions d'ions lourds.

2 II y aura environ 10 petabytes (soit $10^{16}$ bytes) par an de données. 